dungen, so wie dem Wasser so vollständig entzogen werden, dass nach dem Durchgange ihrer Lösungen durch Schichten, die nicht höher als die gewöhnliche Ackerkrume sind, die chemische Analyse kaum Spuren von diesen Stoffen mehr nachzuweisen vermag, so lässt es sich nicht denken, dass Regenwasser für sich oder mit Hülfe von wenigen Procenten Kohlensäure das Vermögen besitzt, diese Stoffe der Ackerkrume zu entziehen und eine im Boden fortbewegbare Lösung zu bilden, dass also unsere Culturgewächse ihre wichtigsten und zum Wachsthum wesentlichsten mineralischen Bestandtheile aus einer Lösung vom Boden empfangen können. Die Salze bleiben nur dann aufgelöst, wenn der Boden, den sie durchdringen, bereits mit ihnen gesättigt ist, und können nur in diesem Falle durch Regenwasser hinweg. geführt werden. Diese Stoffe, wolche das Regenwasser aus der Ackerkrume aufzulösen vermag, sind in dem Drainwasser enthalten, sie bleiben aber weit hinter der Menge zurück, welche sich in der Asche der Pflanzen vorfinden, wie deutlich die Liebig'schen Berechnungen geben. Die Aufnahme von Mineralbestandtheilen muss deshalb durch irgend eine Kraft der Pflanze erfolgen, durch eine in ihren Wurzeln thätig mitwirkende Ursache, wodurch das die Wurzel umgebende Wasser erst das Vermögen empfängt, gewisse Mineralbestandtheile zu lösen, die es für sich allein nicht auflöst.

Anders verhält es sich mit den Wasserpflanzen, deren Wurzeln den Boden nicht berühren; sie müssen ihre mineralische Nahrung aus dem umgebenden Medium nehmen, aber keineswegs in den Verhältnissen, in denen sie ihnen von der Flüssigkeit dargeboten werden, sondern nach ihrer eignen Auswahl, wie auch aus den Analysen von Lemna trissulea und von dem Wasser, in dem diese Pflanze wuchs, hervorging. (Annal.der Chem. u. Pharm. $X X V .109$ - 144.) $G$.

\title{
Untersuchungen über die Amidsäuren.
}

Bei Vergleichung der aus Benzoësäure gebildeten Benzamsäure mit dem Glycocoll war Cah ours erstaunt iuber die Aehnlichkeit der Eigenschaften beider Körper. Diese merkwürdige Substanz vereinigt sich wie das Glycocoll sowohl mit Säuren, als auch mit Basen zu bestimmten, schön krystallisirenden Verbindungen, wie das Gerland 
beobachtete, dem man die Entdeckung dieser interessanten Producte verdankt. Obendrein besitzt die Mehrzahl dieser Verbindungen den schwach süssen Geschmack, den man auch bei den Glycocollverbindungen bemerkt. Cahours versuchte deshalb, mit der Benzamsäure auch andere Säuren, als Schwefelsäure und Salpetersäure zu vereinigen, und es gelang ihm, solche salzartige Verbindungen der Benzamsäure mit Phosphorsäure, Oxalsäure, Salzsäure, Bromwasserstoff u. s. w. hervorzubringen. Weiter gelang es ihm, die genannten unorganischen Säuren auch mit den der Benzamsäure homologen Säuren, nämlich mit Toluamsäure, Cuminamsäure und Anisamsäure zu krystallisirenden Salzen zu vereinigen. Cahours wurde hierdurch zu dem Versuch geführt, das Glycocoll künstlich darzustellen und es gelang ihm dieser Versuch. Das Glycocoll ist eben Acetamsäure. Die folgende Tafel erläutert die Beziehungen dieser merkwürdigen basischen Säuren oder sauren Basen zu ihren Stammsäuren.

Formylreihe.

$\mathrm{C}^{4} \mathrm{H}^{4} \mathrm{O}^{4}=$ Essigsäure. $\quad \mathrm{C}^{4} \mathrm{H}^{3}\left(\mathrm{H}^{2} \mathrm{~N}\right) \mathrm{O}^{4}=$ Glycocoll. $\mathrm{C}^{6} \mathrm{H}^{6} \mathrm{O}^{4}=$ Propionsäure. $\mathrm{C}^{6} \mathrm{H}^{5}\left(\mathrm{H}^{2} \mathrm{~N}\right) \mathrm{O}^{4}=$ Alanin. $\mathrm{C}^{12} \mathrm{H}^{12} \mathrm{O}^{4}=$ Capronsäure. $\mathrm{C}^{12} \mathrm{H}^{11}\left(\mathrm{H}^{2} \mathrm{~N}\right) \mathrm{O}^{4}=$ Leucin.

Benzoylreihe.

$\mathrm{C}^{14 \mathrm{H}^{6}} \mathrm{O}^{4}=$ Benzoësäure. $\mathrm{C}^{14} \mathrm{H}^{5}\left(\mathrm{H}^{2} \mathrm{~N}\right) \mathrm{O}^{4}=$ Benzamsäure. $\mathrm{C}^{16} \mathrm{H}^{8} \mathrm{O}^{4}=$ Toluylsäure. $\mathrm{C}^{16 \mathrm{H}^{7}}\left(\mathrm{H}^{2} \mathrm{~N}\right) \mathrm{O}^{4}=$ Toluamsäure. $\mathrm{C}^{20} \mathrm{H}^{12} \mathrm{O}^{4}=$ Cuminsäure. $\mathrm{C}^{20} \mathrm{H}^{11}\left(\mathrm{H}^{2} \mathrm{~N}\right) \mathrm{O}^{4}=$ Cuminamsäure.

Hiernach ist also Glycocoll $=$ Acetamsäure, Alanin $=$ Propiamsäure und Leucin = Caproamsäure.

\section{Salze der Benzamsäure.}

Salzsaure Benzamsäure $=\mathrm{C}^{14} \mathrm{H}^{7} \mathrm{NO}^{4}, \mathrm{HCl}$. Man löst reine Benzamsäure in überschüssiger siedender Salzsäure, fügt etwas Alkohol zu und lässt erkalten. Das Salz scheidet sich in feinen farblosen Nadeln aus. Diese sind wenig löslich in salzsäurehaltigem Wasser, ziemlich löslich in reinem Wasser und im Weingeist. Aether scheidet aus der alkoholischen Lösung nichts ab. Das Salz schmilzt bei gelinder Wärme. Platinchlorid giebt damit eine krystallisirbare, im Alkohol ziemlich lösliche Verbindung. $\mathrm{C}^{14} \mathrm{H}^{7} \mathrm{NO}^{4}, \mathrm{HCl}+\mathrm{PtCl}^{2}$, goldgelbe Nadeln von Seidenglanz. Auch Quecksilberchlorid giebt damit eine krystallisirbare Verbindung.

Bromwasserstoff-Benzamsä ure $=\mathrm{C}^{14} \mathrm{H}^{7} \mathrm{NO}^{4}$, HBr. Feine Nadeln, der salzsauren Verbindung sehr 
ähnlich. Wenig löslich im mit $\mathrm{HBr}$ gesättigten Wasser, leicht löslich im reinen Wasser und im Alkohol.

Die Aethranilsäure, isomer mit Benzamsäure, liefert nach Cahours mit den Säuren ebenso schön krystallisirende Salze, wie die Benzamsäure. Nach $\mathrm{K}$ üb el (Ann. der Chem. u. Pharm. Bd. CII. S. 236) liefert die Aethranilsäure mit Salzsäure ein Salz $=\mathrm{C}^{14} \mathrm{H}^{7} \mathrm{NO}^{4}, \mathrm{HCl}$ in nadelförmigen Krystallen, mit Salpetersäure grosse Prismen von $\mathrm{C}^{14} \mathrm{H}^{7} \mathrm{NO}^{4}, \mathrm{HO}, \mathrm{NO}^{5}$, mit Oxalsäure die Verbindung $\mathrm{C}^{14} \mathrm{H}^{7} \mathrm{NO}^{4}, \mathrm{HO}, \mathrm{C}^{2} \mathrm{O}^{3}$ und mit Schwefelsäure das Salz $\mathrm{C}^{14} \mathrm{H}^{7} \mathrm{NO}^{4}, \mathrm{HO}, \mathrm{SO}^{3}$.

Aetherbenzamsäure $=\mathrm{C}^{4} \mathrm{H}^{5} \mathrm{O}, \mathrm{C}^{14} \mathrm{H}^{6} \mathrm{NO}^{3}=$ $\mathrm{C}^{18} \mathrm{H}^{11} \mathrm{NO}^{4}=\mathrm{C}^{14} \mathrm{H}^{4}\left(\mathrm{C}^{4} \mathrm{H}^{5}, \mathrm{H}^{2} \mathrm{~N}\right) \mathrm{O}^{4}$. Nitrobenzoësaures Aethyloxyd, durch Schwefelammonium reducirt, liefert eine röthliche Flüssigkeit, unreine Aetherbenzamsäure. Um sie von anhängendem Nitrobenzoësäure-Aether zu reinigen, kocht man sie mit Salzsäure, worin sie sich leicht löst, während der Nitrobenzoësäureäther nicht angegriffen wird. Auf Zusatz eines Ueberschusses von Ammoniak fällt die Aetherbenzamsäure (der basische Benzamsäureäther) nieder. Man wäscht sie mit Wasser und trocknet sie im Vacuum über $\mathrm{HO}, \mathrm{SO}^{3}$.

Die Aetherbenzamsäure ist eine Flüssigkeit, unlöslich in alkalischen Lösungen, leicht löslich in Säuren zu wohl krystallisirten Salzen. Einige derselben sind zerfliesslich. Die Aetherbenzamsäure besitzt sehr ausge. sprochene basische Eigenschaften. Sie ist kaum löslich im Wasser, leicht löslich im Alkohol und Aether, nach deren Verdunstung sie in Form eines Oeles zurückbleibt. Sie wird von kalter concentrirter Kalilauge nicht :ngegriffen; von siedender Kalilauge wird sie zersetzt, unter Entwickelung von Alkohol und Bildung von benzamsaurem Kali. Mit wässerigem Ammoniak übergossen versehwindet sie völlig unter Bildung des Amids der Benzamsäure, d. h. Phenylharnstoffs $\mathrm{C}^{14} \mathrm{H}^{4}\left(\mathrm{C}^{4} \mathrm{H}^{5}, \mathrm{H}^{2} \mathrm{~N}\right) \mathrm{O}^{4}$ $+\mathrm{H}^{3} \mathrm{~N}=\mathrm{C}^{2} \mathrm{H}^{3}\left(\mathrm{C}^{2} \mathrm{H}^{5}\right) \mathrm{N}^{2} \mathrm{O}^{2}+\mathrm{C}^{4} \mathrm{H}^{6} \mathrm{O}^{2}$ Aetherbenzamsäure + Ammoniak $=$ Phenylharnstoff + Alkohol.

Salzsaure Aetherbenzamsäure $=\mathrm{C}^{18} \mathrm{H}^{11} \mathrm{NO}^{4}$, HCl. Entsteht durch unmittelbare Vereinigung der Aetherbenzamsäure mit Salzsäure. Farblose Krystalle. Giebt mit Platinchlorid eine krystallisirbare, orangegelbe, schwer lösliche Verbindung $\mathrm{C}^{18} \mathrm{H}^{11} \mathrm{NO}^{4}, \mathrm{HCl}+\mathrm{PtCl}^{2}$.

Salpetersaure Aetherbenzamsäure $=$ $\mathrm{C}^{18} \mathrm{H}^{11} \mathrm{NO}^{4}, \mathrm{HO}, \mathrm{NO}^{5}$. Zarte Prismen, leicht löslich im 
Wasser, Alkohol und Aether. Unzersetzt schmelzbar; bei stärkerer Hitze sich zersetzend.

Methyloxydbenzamsäure $=\mathrm{C}^{2} \mathrm{H}^{3} \mathrm{O}, \mathrm{C}^{14} \mathrm{H}^{6} \mathrm{NO}^{3}$ $=\mathrm{C}^{16} \mathrm{H}^{9} \mathrm{NO}^{4}=\mathrm{C}^{14} \mathrm{H}^{4}\left(\mathrm{C}^{2} \mathrm{H}^{3}, \mathrm{H}^{2} \mathrm{~N}\right) \mathrm{O}^{4}$. Nitrobenzonsaures Methyloxyd, mit Schwefelammonium behandelt, liefert ein braunes Oel, welches bei der Reinigung farblos wird; dieses ist Methyloxydbenzamsäure. Sie bildet mit den Säuren bestimmte Salze, die sehr leicht löslich sind und häufig sehr zerfliesslich.

Toluamsäure $=\mathrm{C}^{16} \mathrm{H}^{9} \mathrm{NO}^{4}$. Entsteht nach Bouilhet bei Einwirkung des Schwefelammoniums auf Nitrotoluylsïure.

Salzsaure Toluamsäure $=\mathrm{C}^{16} \mathrm{H}^{9} \mathrm{NO}^{4}, \mathrm{HCl}$, bildet perlmutterglänzende Nadeln, wenig löslich in kaltem, salzsäurehaltigem Wasser, leicht löslich im heissen Wasser und im Weingeist. Platinchlorid giebt damit rothbraune Nadeln von $\mathrm{C}^{16} \mathrm{H}^{9} \mathrm{NO}^{4}, \mathrm{HCl}, \mathrm{PtCl}^{2}$.

$\mathrm{Cum}$ in ams äure $=\mathrm{C}^{20} \mathrm{H}^{13} \mathrm{NO}^{4}=\mathrm{C}^{20} \mathrm{H}^{11}\left(\mathrm{H}^{2} \mathrm{~N}\right) \mathrm{O}^{4}$. Entsteht bei Einwirkung des Schwefelammoniums auf Nitrocuminsäure. Farblose Krystalle, wenig löslich in kaltem, weit löslicher in heissem Wasser, in Alkohol und Aether. Die Krystalle sind oft tafelartig und gleichen der Cuminsäure. Die Cuminamsäure verbindet sich mit Basen und Säuren zu krystallisirbaren Salzen. Mit wasserfreiem Baryt, oder Kalihydrat destillirt, zerlegt sie sich unter Bildung von kohlensaurem Alkali und Destillation von Cumidin $\mathrm{C}^{18} \mathrm{H}^{13} \mathrm{~N}$, welches sich zu einem ammoniakalisch aromatischem Oele verdichtet und mit Platinchlorid eine krystallisirbare Verbindung eingeht. $\mathrm{C}^{20} \mathrm{H}^{13} \mathrm{NO}^{4}+2 \mathrm{BaO}=\mathrm{C}^{18} \mathrm{H}^{13} \mathrm{~N}+2\left(\mathrm{BaO}^{3} \mathrm{CO}^{2}\right)$.

Salzsaure Cuminamsäure $=\mathrm{C}^{20} \mathrm{H}^{13} \mathrm{NO}^{4}, \mathrm{HCl}$. Zarte Nadeln, ziemlich löslich in reinem Wasser; Salzsäure scheidet aus dieser Lösung einen Theil des Salzes ab. Platinchlorid giebt damit eine Verbindung $\mathrm{C}^{20} \mathrm{H}^{13} \mathrm{NO}^{4}$, $\mathrm{HCl}+\mathrm{PtCl}^{2}$ in ziemlich grossen röthlichen Nadeln, die sich im heissen Weingeist lösen.

$\mathrm{Sch}$ efels aure Cum in amsäure $=\mathrm{C}^{20} \mathrm{H}^{13} \mathrm{NO}^{4}$, $\mathrm{HO}, \mathrm{SO}^{3}$ : Seideglänzende Prismen, wenig löslich in kaltem, leicht löslich in siedendem Wasser. Von süsslichem Geschmack. Prismen.

Salpetersaure Cuminamsäure bildet schöne

Darstellung der Cuminamsäure. Man erhitzt Nitrocuminsäure mit Schwefelammonium, verjagt das überschüssige Ammoniak durch Concentration der Flüssigkeit 
bei gelinder Wärme, trennt den ausgeschiedenen Schwefel durch Filtration, zerlegt das concentrirte Filtrat durch Essigsäure im geringen Ueberschuss, wäscht den erhaltenen Niederschlag mit kaltem Wasser, trocknet denselben und krystallisirt aus alkoholischer Lösung.

Oxycuminsäure $=\mathrm{HO}, \mathrm{C}^{20} \mathrm{H}^{11} \mathrm{O}^{5}=\mathrm{C}^{20} \mathrm{H}^{12} \mathrm{O}^{6}$. Bildet sich bei Einwirkung von Stickoxydgas auf eine Lösung der Cuminamsäure in Salpetersäure von mittlerer Stärke.

Sie krystallisirt in kleinen Prismen von bräunlichgelber Farbe, löst sich wenig in kaltem, leichter in heissem Wasser und im Alkohol. Liefert mit Basen wohlkrystallisirte Salze. Oxycuminsaures Silberoxyd $=\mathrm{AgO}$, $\mathrm{C}^{20} \mathrm{H}^{11} \mathrm{O}^{5}$. Sie ist der Oxybenzoësäure $\mathrm{C}^{14} \mathrm{H}^{6} \mathrm{O}^{6}$ und der Glycolsäure $\mathrm{C}^{4} \mathrm{H}^{4} \mathrm{O}^{6}$ analog.

Aethercuminamsäure $=\mathrm{C}^{4} \mathrm{H}^{5} \mathrm{O}, \mathrm{C}^{20} \mathrm{H}^{12} \mathrm{NO}^{3}$ $=\mathrm{C}^{24} \mathrm{H}^{17} \mathrm{NO}^{4}=\mathrm{C}^{20} \mathrm{H}^{10}\left(\mathrm{C}^{4} \mathrm{H}^{5}, \mathrm{H}^{2} \mathrm{~N}\right) \mathrm{O}^{4}$. Nitrocuminsäureäther in alkoholischer Lösung wird durch Schwefelammonium rasch reducirt; beim Abdampfen der Flüssigkeit scheidet sich reichlich Schwefel aus. Das Filtrat setzt beim Verdunsten eine ölige Flüssigkeit von dunkler Farbe ab, welche durch Lösen in Weingeist und Fällung mit Wasser gereinigt, die Aethercuminamsäure darstellt. Ein schweres Oel, löslich in $\mathrm{HCl}, \mathrm{HBr}, \mathrm{NO}^{5}, \mathrm{SO}^{3}$, mit denen es sehr lösliche, krystallisirbare Salze bildet. Ammoniak bildet damit ein Amid, wahrscheinlich eine dem Phenylharnstoff analoge Verbindung.

Anisamsäure $=\mathrm{C}^{16} \mathrm{H}^{9} \mathrm{NO}^{6}=\mathrm{C}^{16} \mathrm{H}^{7}\left(\mathrm{H}^{2} \mathrm{~N}\right) \mathrm{O}^{6}$. Eine wässerige Lösung des nitroanissauren Ammoniaks wird durch Sättigung mit Schwefelwasserstoffgas rasch reducirt, besonders bei gelinder Erwärmung. Es scheidet sich Schwefel ab und in der Lösung findet sich jetzt anisamsaures Ammoniak. Man concentrirt die Flüssigkeit und vermischt sie im geringen Ueberschuss mit Essigsäure. Es fällt die Anisamsäure als bräunliche Masse, die man auf dem Filter mit Wasser wäscht. Man löst in siedendem Weingeist und erhält beim Erkalten der Lösung Krystalle von Anisamsäure, die durch Umkrystallisiren gereinigt werden. Die Anisamsäure bildet bernsteinfarbene Krystalle, die bei gelinder Wärme zu klarer Flüssigkeit schmelzen; beim Abkühlen der Flüssigkeit erstarrt dieselbe krystallinisch. Bei starker Hitze wird die Säure zerstört. Wasser löst nur wenig, Alkohol und Aether lösen viel derselben. Die Anisamsäure löst sich sehr leicht in alkalischen und sauren Flüssig- 
keiten und liefert mit Alkalien und mit Säuren krystallisirende Salze.

Mit überschüssigem Baryt oder mit Kalihydrat destillirt, liefert sie kohlensaures Alkali und die flüchtige Basis Anisidin.

$\mathrm{C}^{16} \mathrm{H}^{9} \mathrm{NO}^{6}+2 \mathrm{BaO}=\mathrm{C}^{14} \mathrm{H}^{9} \mathrm{O}^{2} \mathrm{~N}+2\left(\mathrm{BaO}, \mathrm{CO}^{2}\right\rangle$ Anisamsäure + Baryt $=$ Anisidin + kohlens. Baryt.

Löst man Anisamsäure in mässig starker Salpetersäure und leitet längere Zeit Stickoxydgas durch die Lösung, so entweicht Stickgas und in Lösung bleibt stickstofffreie Oxanissäure.

Salzsaure Anisamsäure $=\mathrm{C}^{16} \mathrm{H}^{9} \mathrm{NO}^{6}, \mathrm{HCl}$ bildet Krystalle, löslich im Wasser und Weingeist; wenig löslich im Aether, kaum löslich in salzsaurem Wasser. Platinchlorid giebt damit $\mathrm{C}^{16} \mathrm{H}^{9} \mathrm{NO}^{6}, \mathrm{HCl}+\mathrm{PtCl}^{2}$, bräunliche Krystalle, ziemlich löslich in heissem Wasser und Weingeist.

Schwefelsaure Anisamsäure $=\mathrm{C}^{16} \mathrm{H}^{9} \mathrm{NO}^{6}$, $\mathrm{HO}, \mathrm{SO}^{3}$. Seideglänzende Nadeln, von süssem Geschmack, leicht löslich im Wasser und in siedendem Weingeist.

Salpetersaure Anisamsäure $=\mathrm{C}^{16} \mathrm{H}^{9} \mathrm{NO}^{6}$, HO, NO5. Locker, büschelweise vereinigte Prismen, reichlich löslich im Wasser und Weingeist. Salpetersäure vermindert sehr die Löslichkeit derselben im Wasser.

Aetheranisamsäure = Anisamäther $=\mathrm{C}^{4} \mathrm{H}^{5} \mathrm{O}$, $\mathrm{C}^{16} \mathrm{H}^{8} \mathrm{NO}^{5}=\mathrm{C}^{20} \mathrm{H}^{13} \mathrm{NO}^{6}=\mathrm{C}^{16} \mathrm{H}^{6}\left(\mathrm{C}^{4} \mathrm{H}^{5}, \mathrm{H}^{2} \mathrm{~N}\right) \mathrm{O}^{6}$. Nitroanissäureäther in weingeistiger Lösung wird durch Schwefelammonium rasch zu Aetheranisamsäure reducirt. Beim Abdampfen der Lösung scheidet sich Schwefel aus und das Filtrat liefert beim weiteren Eindunsten ein dickes Oel, das bald krystallinisch gesteht. Farblose Prismen, leicht löslich in siedendem Alkohol, weniger leicht im Aether. Nicht löslich in Kali-, Natron- und Ammoniakflüssigkeit; die Aetheranisamsäure giebt keine Verbindungen mit den Alkalien. (Der Name "Säure" ist deshalb nicht passend für dieselbe.) Mit Salzsäure, Bromwasserstoff, Salpetersäure und Schwefelsäure liefert sie krystallisirbare Salze. Das salpetersaure Salz verändert sich rasch unter Schwärzung.

Die Aetheranisamsäure (oder Anisamäther) löst sich leicht in siedender wässeriger Oxalsäure; beim Erkalten scheidet sich das oxalsaure Salz des Anisamäthers in warzig gehäuften Krystallen aus. Weinsäure bildet mit Anisamäther ebenfalls ein krystallisirbares Salz. Die Aufösung aller dieser Salze wird durch Ammoniak oder 
Kalilauge zersetzt und die Anisamäther gefällt. (Analog den Alkaloiden.)

Salzsaurer Anisamäther $=\mathrm{C}^{20} \mathrm{H}^{13} \mathrm{NO}^{6}, \mathrm{HCl}$. Anisamäther vereinigt sich unmittelbar mit Salzsäure. Das farblose krystallinische Salz ist wenig löslich in kaltem, leicht löslich in heissem Wasser, ziemlich löslich im Aether, sehr löslich im Alkohol. Schmilzt in der Wärme, zersetzt sich in der Hitze. Platinchlorid giebt damit $\mathrm{C}^{20} \mathrm{H}^{13} \mathrm{NO}, \mathrm{HCl}-\mathrm{PtCl}^{2}$; röthlich-braune Prismen, die in der Luft bald undurchsichtig werden.

Methyloxydanisamsäure $=$ Anisammethyläther $=\mathrm{C}^{2} \mathrm{H}^{3} \mathrm{O}, \mathrm{C}^{16} \mathrm{H}^{8} \mathrm{NO}^{5}=\mathrm{C}^{18} \mathrm{H}^{11} \mathrm{NO}^{6}=\mathrm{C}^{16} \mathrm{H}^{6}$ $\left(\mathrm{C}^{2} \mathrm{H}^{3}, \mathrm{H}^{2} \mathrm{~N}\right) \mathrm{O}^{6}$ entsteht aus nitroanissaurem Methyloxyd durch Einwirkung des Schwefelammoniums. Krystallisirbar. Unfähig, sich mit Basen zu vereinigen, liefert der Anisammethyläther mit Säuren krystallisirbare Salze. Leicht löslich im Alkohol und Aether.

Salzsaurer Anisammethyläther bildet zarte, glänzende Prismen, wenig löslich in kaltem Wasser, leicht löslich in siedendem Wasser und im Weingeist. Mit Platinchlorid liefert er die Verbindung $\mathrm{C}^{18} \mathrm{H}^{11} \mathrm{NO}^{6}, \mathrm{HCl}+$ $\mathrm{PtCl}^{2}$ als röthliche, durchsichtige Prismen, die an trockner Luft rasch undurchsichtig werden.

Acetamsäure oder Glycocoll $=\mathrm{C}^{4} \mathrm{H}^{3}\left(\mathrm{H}^{2} \mathrm{~N}\right) \mathrm{O}^{4}$ $=\mathrm{C}^{4} \mathrm{H}^{5} \mathrm{NO}^{4}$. Man erhitzt Monochloressigsäure $\mathrm{C}^{4} \mathrm{H}^{3} \mathrm{ClO}^{4}$ mit einer weingeistigen Aetzammoniakflüssigkeit. Es entsteht Salmiak und Glycocoll. Schöne Prismen, fähig, sowohl mit Silberoxyd, als auch mit Salzsäure und Salpetersäure krystallisirte Verbindungen einzugehen.

Man weiss, dass Glycocoll mit Benzoësäure die Hippursäure (Benzursäure), mit Salicylsäure die Salicylursäure bildet. Cahours stellte nun durch Einwirkung des Chlorcumyls und Chloranisyls auf Glycocoll-Silberoxyd, die der Hippursäure analogen Säuren Cuminursäure und Anisursäure dar. Die krystallisirte Cuminursäure $=\mathrm{C}^{24} \mathrm{H}^{15} \mathrm{NO}^{6}=\mathrm{C}^{4} \mathrm{H}^{2}\left(\mathrm{H}^{2} \mathrm{~N}\right) \mathrm{O}^{3}, \mathrm{C}^{20} \mathrm{H}^{11} \mathrm{O}^{3}$. Mit Salzsäure gekocht liefert sie unter Wasseraufnahme Glycocoll und Cuminsäure.

Die Anisursäure $=\mathrm{C}^{20} \mathrm{H}^{11} \mathrm{NO}^{8}$ bildet farblose Prismen, durch Hitze zerstörbar und durch Kochen mit Säuren Glycocoll und Anissäure liefernd. Das Silbersalz = $\mathrm{AgO}, \mathrm{C}^{20} \mathrm{H}^{10} \mathrm{NO}^{7}$.

Sch lus b emerkungen. Die beschriebenen Amidsäuren haben eine andere Bildungsweise, als die gewöhnlichen Amide und Imide. Die letzteren entstehen 
durch Ersetzung des Sauerstoffs durch Amid oder Imid, die ersteren durch Ersetzung des Wasserstoffs durch Amid, z. B.

$\mathrm{C}^{14} \mathrm{H}^{6} \mathrm{O}^{6}+\mathrm{H}^{3} \mathrm{~N}=\mathrm{C}^{14} \mathrm{H}^{6} \mathrm{O}^{4}(\mathrm{H} \mathrm{N})+2 \mathrm{HO}$ Salicylsäure + Ammoniak $=$ Salicylimid + Wasser. $\mathrm{C}^{14} \mathrm{H}^{5}\left(\mathrm{NO}^{4}\right) \mathrm{O}^{4}+6 \mathrm{H}=\mathrm{C}^{14} \mathrm{H}^{5}\left(\mathrm{H}^{2} \mathrm{~N}\right) \mathrm{O}^{4}+4 \mathrm{HO}$ Nitrobenzoësäure + Wasserstoff $=$ Benzamsäure + Wasser.

Die besprochenen Amidsäuren spielen gleichzeitig die Rolle von Säuren und von Basen. Mit salpetriger Säure behandelt, verlieren sie ihren sämmtlichen Stickstoff; dieser entweicht in Gasform. Die Säuren nehmen dafür Sauerstoff auf und bilden Säuren, welche 2 Aeq. Sauerstoff mehr enthalten, als die Stammsüuren, z. B. $\mathrm{C}^{14} \mathrm{H}^{7} \mathrm{NO}^{4}+\mathrm{NO}^{3}=\mathrm{N}^{2}+\mathrm{HO}^{2} \mathrm{C}^{14} \mathrm{H}^{6} \mathrm{O}^{6}$ Benzamsäure Oxybenzoësäure. $\mathrm{C}^{4} \mathrm{H}^{5} \mathrm{NO}^{4}+\mathrm{NO}^{3}=\mathrm{N}^{2}+\mathrm{HO}+\mathrm{C}^{4} \mathrm{H}^{4} \mathrm{O}^{6}$ Glycocoll Glycolsäure. $\mathrm{C}^{6} \mathrm{H}^{7} \mathrm{NO}^{4}+\mathrm{NO}^{3}=\mathrm{N}^{2}+\mathrm{HO}+\mathrm{C}^{6} \mathrm{H}^{6} \mathrm{O}^{6}$ Alanin

Mit ätzenden Alkalien bei $300^{\circ} \mathrm{C}$. destillirt, spalten sich die Amidsäuren in Kohlensäure und flüchtige Ammoniakbasen (ammoniaques composées), in ähnlicher Weise, wie die Stammsäuren dabei in Kohlensäure und die entsprechenden Kohlenwasserstoffe zerfallen z. B. $\mathrm{C}^{14} \mathrm{H}^{6} \mathrm{O}^{4}+2 \mathrm{BaO}=2\left(\mathrm{BaO}, \mathrm{CO}^{2}\right)+\mathrm{C}^{12} \mathrm{H}^{6}$ Benzoësäure Phenylwasserstoff. $\mathrm{C}^{14} \mathrm{H}^{5}\left(\mathrm{H}^{2} \mathrm{~N}\right) \mathrm{O}^{4}+2 \mathrm{BaO}=2\left(\mathrm{BaO}, \mathrm{CO}^{2}\right)+\left(\mathrm{C}^{12} \mathrm{H}^{5}, \mathrm{H}^{2} \mathrm{~N}\right)$ Phenylamid.

Schwanert und Limpricht erhielten aus Alanin Aethylamin und aus Leucin Amylamin.

$\mathrm{C}^{6} \mathrm{H}^{7} \mathrm{NO}^{4}+2 \mathrm{BaO}=2\left(\mathrm{BaO}_{2} \mathrm{CO}^{2}\right)+\mathrm{C}^{4} \mathrm{H}^{5}, \mathrm{H}^{2} \mathrm{~N}$ Alanin Aethylamin. $\mathrm{C}^{12} \mathrm{H}^{13} \mathrm{NO}^{4}+2 \mathrm{BaO}=2\left(\mathrm{BaO}, \mathrm{CO}^{2}\right)+\mathrm{C}^{10} \mathrm{H}^{11}, \mathrm{H}^{2} \mathrm{~N}$ Leucin Amylamin.

Nach Cahours giebt das wasserfreie Glycocoll mit wasserfreiem Baryt destillirt Methylamin und als. Zersetzungsproduct des letzteren auch Ammoniak.

$\mathrm{C}^{4} \mathrm{H}^{5} \mathrm{NO}^{4}+2 \mathrm{BaO}=2\left(\mathrm{BaO}, \mathrm{CO}^{2}\right)+\mathrm{C}^{2} \mathrm{H}^{3}, \mathrm{H}^{2} \mathrm{~N}$ Glycocoll Methylamin.

Mit Kalihydrat erhitzt, liefert das Glycocoll nur Ammoniak, oxalsaures Kali und Wasserstoff; als Zersetzungsproduct des oxalsauren Kalis auch kohlensaures Kali. 
$\mathrm{C}^{2} \mathrm{H}^{5} \mathrm{~N}+\mathrm{KO}+3 \mathrm{HO}=\mathrm{KO}, \mathrm{C}^{2} \mathrm{O}^{3}+\mathrm{H}^{3} \mathrm{~N}+5 \mathrm{H}$ Methylamin oxalsaures Kali.

(Annal. de Chim. et de Phys. 3. Sér. Juill. 1858. Tóm. LIII. pag. 322 - 359.)

Dr. H. Ludwig.

\section{Ueber eine neue Methode zur quantitativen Bestim- mung der Blausäure.}

Buignet hat ein neues Titrirverfahren zur quantitativen Bestimmung der Blausäure angegeben, welches auf den Eigenthümlichkeiten zweier Kupferverbindungen beruht, auf der Farblosigkeit der Doppelverbindung der Cyanverbindung des Cyankupfers mit dem Cyanammonium einerseits, und andererseits auf der tiefblauen Farbe des schwefelsauren Kupferoxyd-Ammoniaks.

Die Theorie, die Buignet aufstellt, ist folgende: Denken wir uns eine blausäurehaltige Flüssigkeit, die mit überschüssigem Aetzammoniak versetzt worden war, so haben wir darin nothwendig, ausser freiem Ammonials auch Cyanammonium; fuigen wir nun $\mathrm{zu}$ derselben so lange eine titrirte Auflösung von Kupfervitriol hinzu, bis die Anfangs sogleich wieder verschwindende blaue Färbung eine bleibende zu werden beginnt, so giebt uns die eintretende Reaction zu erkennen, dass die zugesetzte Menge Kupfervitriol hingereicht hat, um alles Cyanaumonium zu binden. $\mathrm{Da}$ wir aber eine titrirte Lösung von schwefelsaurem Kupferoxyd angewendet haben, so giebt uns dieselbe unmittelbar den Blausäuregehalt der Flüssigkeit in Procenten an.

Wiewobl zu erwarten war, dass diese dem Principe nach vollkommen richtige Methode sich auch in der Praxis bewähren würde, so ist dieselbe von A. Ferrein einer Prüfung unterworfen worden und dabei folgendermaassen verfahren.

In einer abgewogenen Menge einer officinellen Blausäure wurde der Gehalt an $\mathrm{HCy}$ mittelst salpetersaurem Silberoxyd bestimmt; 3,062 Grm. derselben gaben 0,121 Grm. Cyansilber, worin 0,024 Grm. Cyanwasserstoff, mithin enthielt die angewandte Blausäure nach diesem Versuche 0,795 Proc. $\mathrm{HCy}$.

Alsdann wurde nach Buignet's Angabe eine titrirte Lösung von schwefelsaurem Kupferoxyd bereitet, von welcher $1 / 10$ C.C. genau 0,001 Grm. $\mathrm{H} \mathrm{Cy}$ entsprach. Nach Proportion 2 Aeq. $\mathrm{HCy}: 1$ Aeq. $\mathrm{CuO}, \mathrm{SO}^{3}+5 \mathrm{HO}$

$$
675: 1558=1000: x ; x=2308 \text {, }
$$

Arch. d. Pharm. CXXXXIX. Bds. 3. Hft. 